\title{
Biocomputing Security System: Concatenated Enzyme-Based Logic Gates Operating as a Biomolecular Keypad Lock
}

\author{
Guinevere Strack, Maryna Ornatska, Marcos Pita and Evgeny Katz* \\ Department of Chemistry and Biomolecular Science, Clarkson University, \\ Potsdam NY 13699-5810, USA
}

* To whom all correspondence should be addressed:

Fax: 1-315-2686610; Tel.: 1-315-2684421; E-mail: ekatz@clarkson.edu

\section{Experimental Section:}

Chemicals and reagents

All enzymes and most chemicals were purchased from Sigma-Aldrich and used as supplied. The enzymes that were used are: invertase (Inv) from (S. cerevisiae) (E.C. 3.2.1.26), glucose oxidase (GOx) from Aspergillus niger type X-S (E.C. 1.1.3.4) and microperoxidase-11 (MP-11) prepared by enzymatic degradation of equine heart cytochrome c. All other chemicals (Sigma-Aldrich) were used without further purification: sucrose, 2,2'-azino-bis(3-ethylbenzthiazoline-6-sulphonic acid) (ABTS), 4(2-hydroxyethyl)piperazine-1-ethanesulfonic acid sodium salt (HEPES), 1-ethyl-3-(3dimethylaminopropyl)carbodiimide (EDC), glutaric dialdehyde, aminopropyl)triethoxysilane and toluene (J.T.Baker, HPLC grade). Ultrapure water from NANOpure Diamond (Barnstead) source was used in all of the experiments.

Immobilization of enzymes on a solid support

Glass beads ( $1 \mathrm{~mm}$ diameter, Turtle Rainbow $\left.{ }^{\circledR}\right)$ were used as a solid support for the enzyme immobilization. The surface of the beads was cleaned by reacting with piranha solution, $25 \% \mathrm{v} / \mathrm{v}$ of $\mathrm{H}_{2} \mathrm{O}_{2}(30 \%)$ and $75 \% \mathrm{v} / \mathrm{v}$ of $\mathrm{H}_{2} \mathrm{SO}_{4}(98 \%)$, for 30 minutes, then washed several times with water. (Caution: Piranha is a vigorous oxidant. Piranha solution reacts violently with organic solvents and is a skin irritant. Extreme caution should be exercised when handling piranha solution.) Water was removed from the beads by placing them in a $40{ }^{\circ} \mathrm{C}$ oven overnight. The surface of the glass beads was modified by reacting them with $1 \% \mathrm{v} / \mathrm{v}$ (3-aminopropyl)triethoxysilane (APTES) in toluene 
overnight at room temperature, $23 \pm 2{ }^{\circ} \mathrm{C}$. Then the silanized surface was washed first with toluene and later with ethanol and water to remove unreacted materials. In order to attach covalently Inv or GOx the amino-functionalized surface of the silanized glass beads was reacted with glutaric dialdehyde, $5 \%(\mathrm{v} / \mathrm{v})$ in $0.01 \mathrm{M}$ phosphate buffer, $\mathrm{pH}=7.0$, for 20 minutes at room temperature and then washed several times with the phosphate buffer. The glutaric dialdehyde-activated beads were reacted with Inv or GOx, $1 \mathrm{mg} \mathrm{mL}^{-1}$, in $0.01 \mathrm{M}$ phosphate buffer, $\mathrm{pH}=7.0$, for 20 minutes at room temperature and then washed several times with the phosphate buffer. MP-11, $1 \mathrm{mg} \mathrm{mL}^{-1}$, was covalently bound to the silanized beads in $50 \mathrm{mM}$ HEPES buffer, $\mathrm{pH}=7.2$, in the presence of EDC, $1 \mathrm{mM}$, for 3 hours; then the MP-11-modified beads were washed several times with water to remove unbound materials.

Composition of the biomolecular keypad lock system and the input signals

The biomolecular keypad lock system was composed of $10 \mathrm{mM}$ sucrose, oxygen being in equilibrium with air and $0.1 \mathrm{mM}$ ABTS dissolved in $0.1 \mathrm{M}$ phosphate buffer, $\mathrm{pH}$ 5.0, total reacting volume $1 \mathrm{~mL}$. The biocatalysts, Inv, GOx and MP-11, covalently bound to the glass beads were used as the input signals (A,B,C, respectively) to activate the concatenated AND logic gates. We also performed the similar modification procedure for the immobilization of the biocatalysts on silicon wafers and used AFM to characterize the modified surfaces similarly to the published procedure. ${ }^{1}$ Tapping mode AFM imaging of the enzymes immobilized on silica wafers was performed using a Dimension 3100 (Digital Instruments, Inc., Santa Barbara, CA) microscope in ambient conditions. Silicon tips with a radius of $20 \mathrm{~nm}$, and resonant frequency of $200-300 \mathrm{kHz}$ were used. For the analysis of the AFM data, we used DI nanoscope software. High resolution AFM imaging, Figures SI-1(A) and SI-1(B), show dense surface coverage of Inv and GOx with the individual protein molecules visible as bright white spherical particles. The average diameters of the individual molecules were ca. $8 \mathrm{~nm}$ and ca. $6 \mathrm{~nm}$ for Inv and GOx, respectively, according to the cross-sectional analysis, similar to the expected dimensions of the enzymes derived from the molecular models. ${ }^{2}$ The surface with the immobilized MP-11 layers, Figure SI-1(C), shows high-density coverage; the morphology however points to the presence of molecular aggregates. Under the used immobilization conditions, in the presence of EDC, MP-11 can form aggregates or polymers due to the 
cross-linking of the peptide fragments. These results indicate that Inv and GOx were deposited in a single monolayer containing pinholes between the protein molecules, while MP-11 formed a multilayer assembly on the surface (about 6 layers of MP-11).

The amounts of Inv and GOx immobilized on the glass beads were also experimentally determined using hydrolysis of the protein followed by the optical analysis of the products. ${ }^{3}$ Inv or GOx were immobilized on 2,000 glass beads using APTES and glutaric dialdehyde as previously described. After washing, the beads were submerged in $1 \mathrm{M} \mathrm{NaOH}$ for two hours and then neutralized with hydrochloric acid. The amount of the hydrolyzed protein was estimated using the absorbance at $\lambda=280 \mathrm{~nm}$ and the extinction coefficient of the products obtained upon hydrolysis of the proteins, ${ }^{4}$ E1\% $=16.7$, resulting in approximately 0.35 and 0.40 pmoles per bead for Inv and GOx, respectively. The MP-11 loading was estimated ca. 30 pmoles per bead based on the AFM measurements.

\section{Measurements}

Invertase was immobilized on 2,000 glass beads, 0.7 nmoles Inv, glucose oxidase was immobilized on 1,500 glass beads, 0.6 nmoles GOx, and MP-11 was immobilized on 1,000 glass beads, 30 nmoles MP-11. The beads were exposed to $1 \mathrm{~mL}$ of solution composed of $0.01 \mathrm{M}$ sucrose and $100 \mu \mathrm{M}$ ABTS in $0.01 \mathrm{M}$ phosphate buffer, $\mathrm{pH}=5.0$, for 25 minute intervals before being transferred to a cuvette for optical measurements or being reacted with the next immobilized enzyme. The system composition (concentrations of sucrose and ABTS) and the quantities of the enzyme-inputs were optimized in order to produce a significant change in $|\Delta \mathrm{A}|$, thus resulting in a corresponding "0" or "1" state. The reference cuvette was filled with the same composition as the test cuvette prior to the addition of the enzyme inputs, thus allowing the differential spectra measurements corresponding to the changes in the gate composition originating from the enzyme-induced reactions. The absorbance measurements were performed using a Shimadzu UV-2450PC spectrophotometer. All measurements were done at $23 \pm 2{ }^{\circ} \mathrm{C}$. 

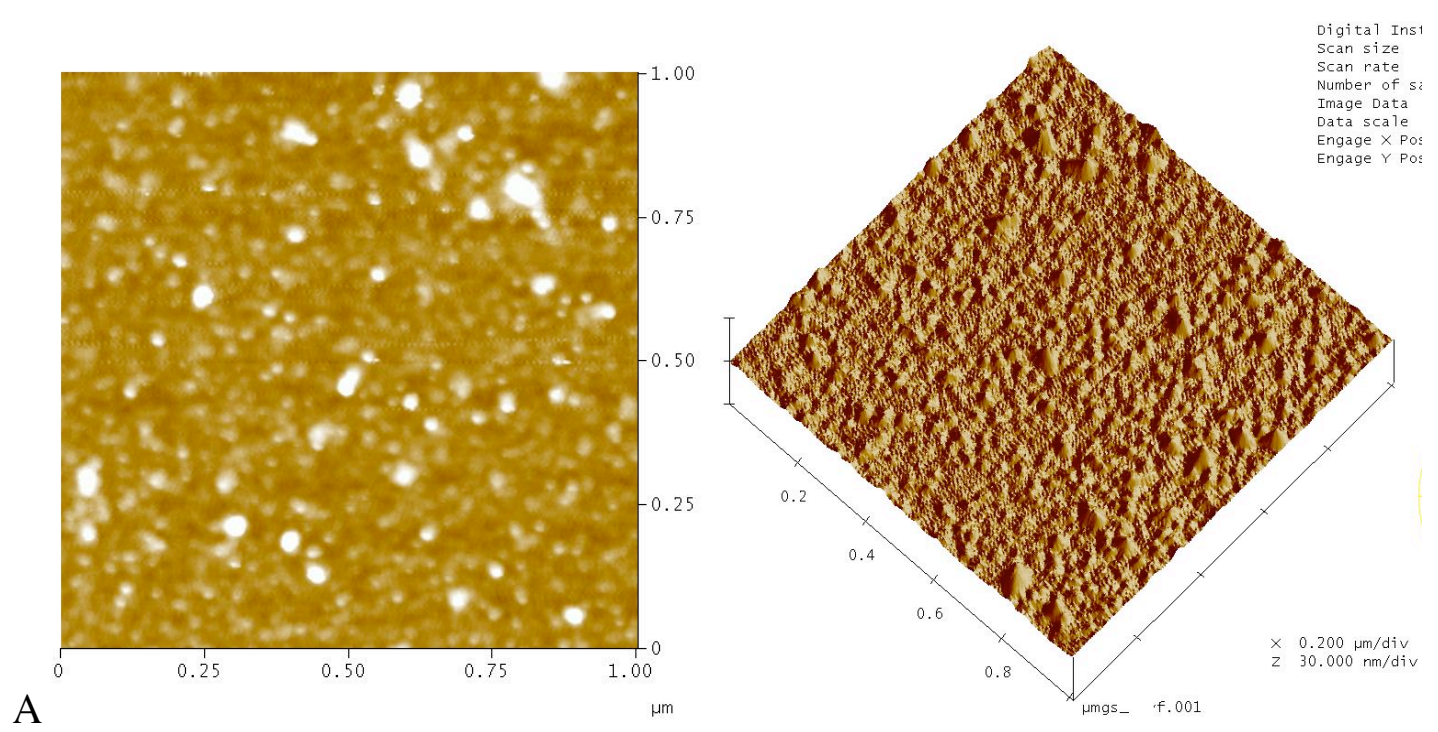

A

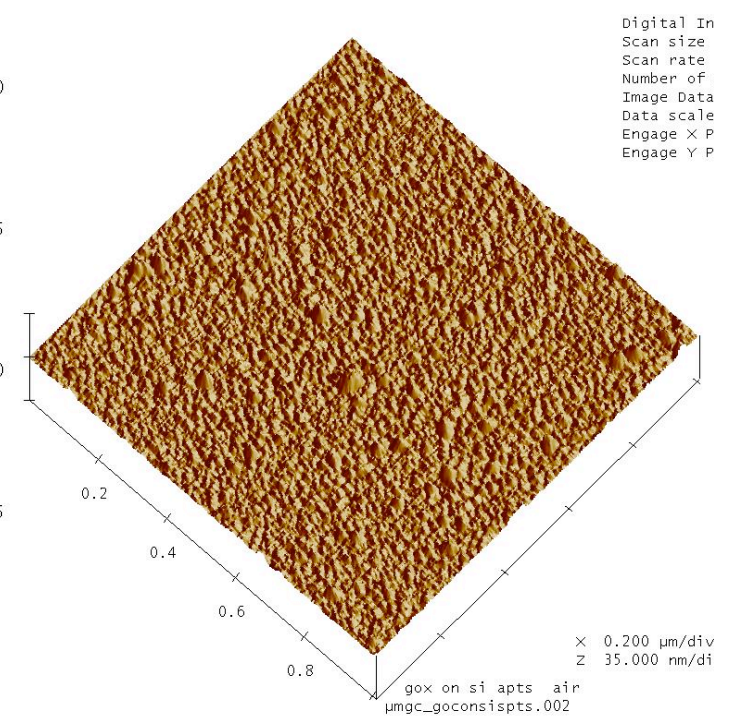

B
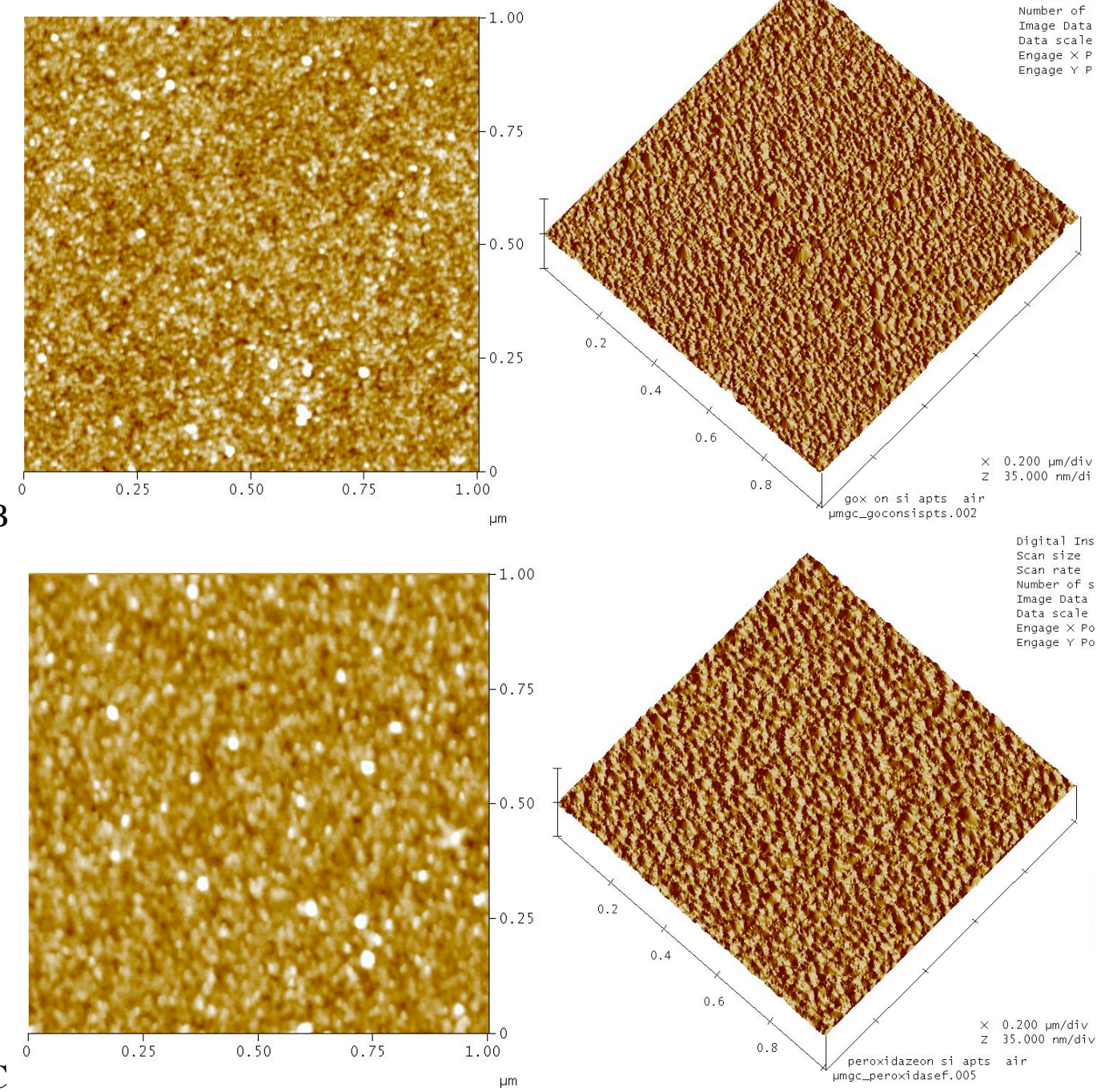

Figure SI-1. AFM topography images of the immobilized enzymes on silica: (A) invertase (Inv), (B) glucose oxidase (GOx), (C) microperoxidase-11 (MP-11). 


\section{References:}

1. Saal, K.; Sammelselg, V.; Lõhmus, A.; Kuusk, E.; Raidaru, G.; Rinken, T.; Rinken, A. Biomolec. Eng. 2002, 19, 195-199.

2. Protein Data Bank: http://www.rcsb.org/pdb/home/home.do

3. H. Sah, J. Pharm. Sci. 1997, 86, 1315-1318.

4. G.D. Fasman, Handbook of Biochemistry and Molecular Biology, CRC Press, Boca Raton, FL, p. 244, 1990. 